\title{
¿Debe cambiar la educación en administración?
}

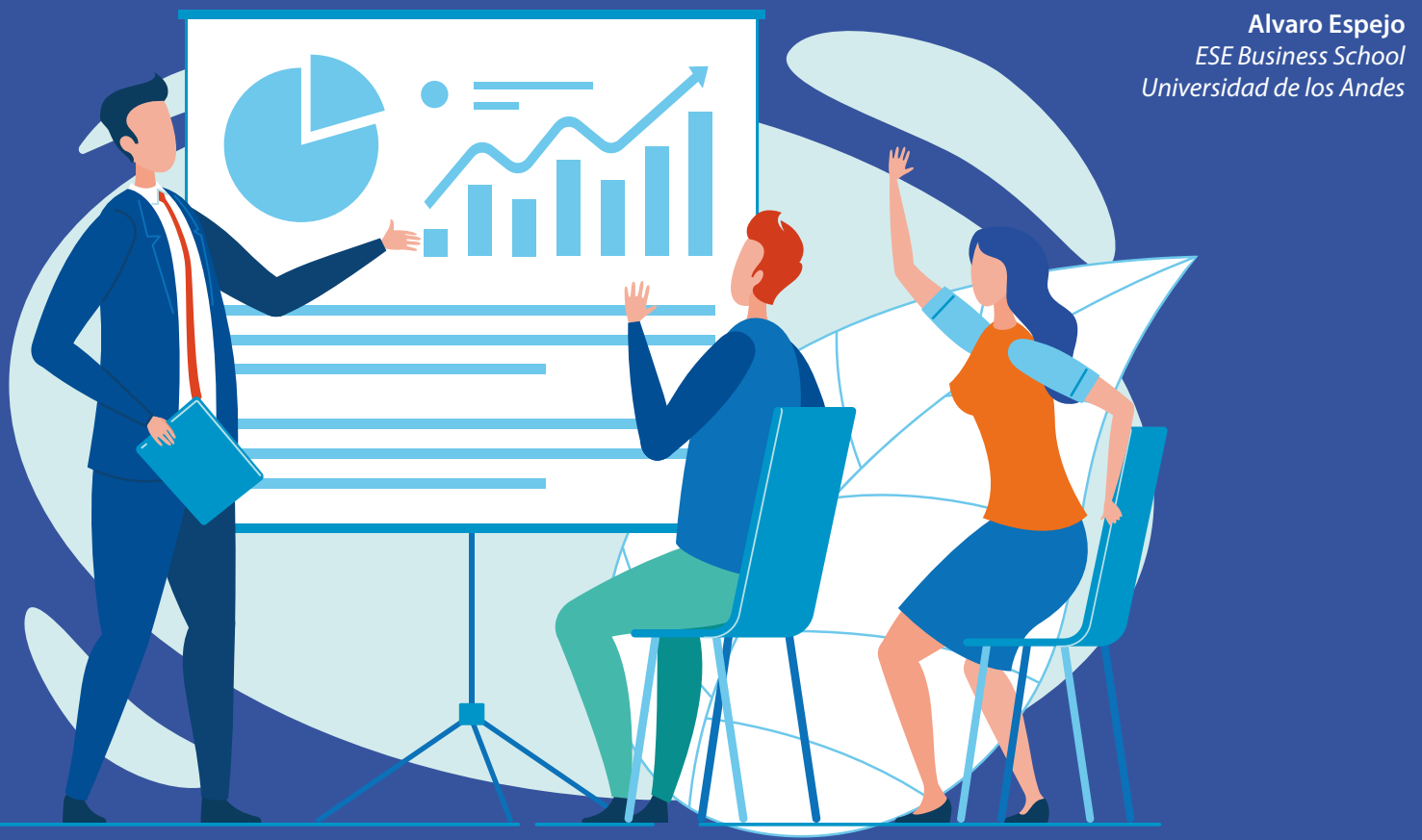

En un año de importantes desafíos y profundos cambios, como ha sido el 2020, es necesario tomarse el tiempo para reflexionar sobre el presente y el futuro de la educación en administración. Aunque para ello es crucial tener una visión crítica, también es fundamental valorar los aportes que se han realizado al campo de la administración a lo largo del tiempo. La investigación nos muestra que las personas, la colaboración y el propósito son los elementos centrales de la organización. Sin embargo, debido a una visión limitada sobre la administración existente en muchas escuelas de negocio, esto muchas veces ha quedado relegado frente a los objetivos económicos de las empresas.

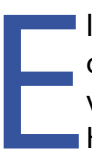

I mundo que estamos viviendo ha obligado a repensar muchas actividades, entre ellas la educación. Ha cambiado la forma de acceder a la educación, han cambiado las competencias requeridas en los educadores $y$, en parte, ha cambiado el foco de la educación, dando más cabida a aspectos psicosociales y bajando en algo la importancia de los contenidos. La educación en negocios también se ha visto afectada. El cambio más visible ha sido el traspaso de programas a formato online. Pero también se pueden observar modificaciones en los contenidos, dando más cabida a temas de personas, relaciones y emociones en la organización. En definitiva, podríamos estar en camino a una visión más humana de la organización. Así y todo, cabe preguntarse si esto es una respuesta a la situación inmediata, y pasada la pandemia volveremos a lo habitual, o si habrá un cambio más profundo y sostenido en el tiempo en la educación en negocios. Una mirada a lo que ha ocurrido anteriormente nos indica que ésta no es la primera crisis que se vive en las escuelas de negocios y que se requiere una reflexión mucho más profunda para que el cambio ocurra.

\section{Supuestos errados y glorificación de la objetividad}

A raíz de algunos escándalos corporativos (como el de ENRON) a inicios de este siglo, Sumantra Ghoshal, académico de la London Business School, realizó un análisis sobre el rol de las universidades y escuelas de economía y administración. En resumen, Ghoshal (2005) argumentaba que la formación que entregan las escuelas de administración favorece la falta de responsabilidad moral de los ejecutivos debido a los supuestos básicos de los modelos usados y a la forma en que se crea conocimiento en la disciplina. El primer argumento se refiere a que los modelos usados en general vienen de la economía, en particular de la escuela de Chicago, y presentan una visión negativa de los seres humanos, caracterizándolo como seres racionales maximizadores de su interés propio y oportunistas. Por ello, 
el rol de las organizaciones y los ejecutivos es evitar el daño que las personas tenderán a hacer (de ahí el extensivo uso de la teoría de agencia y de la economía de costos de transacción). Esta visión se mantiene a pesar de que existe una gran cantidad de evidencia de lo contrario, es decir que las personas se preocupan no sólo de sí mismos, sino también de los demás (Grant, 2008), además de actuar muchas veces en forma no completamente racional, como muestran los ganadores del Premio Nobel Herbert Simon (1978), Daniel Kahneman (2002) y Richard Thaler (2017). El segundo argumento apunta a que, a pesar de que la administración y la economía son ciencias sociales, se busca crear conocimiento siguiendo los métodos de las ciencias naturales, para así aparecer como más objetivos o "científicos". El problema con ello es que mientras los fenómenos de las ciencias naturales (por ejemplo, las estrellas o los compuestos químicos) se comportarán de una forma determinada independiente de la idea preconcebida que tenga el investigador, los seres humanos variarán su comportamiento. Esto se conoce como las profecías autocumplidas. Así, si asumimos que las personas son oportunistas y sólo buscan maximizar su propio beneficio, trataremos a las personas de esa manera, con controles e incentivos en esa línea, y esas personas se empezarán a comportar de esa manera.

\section{Desconexión entre Investigación, Práctica y Enseñanza}

Podemos pensar en múltiples ejemplos de elementos que se enseñan en escuelas de negocios, donde tanto la investigación como la práctica nos han mostrado que no son efectivos. Pablo Fernández, profesor de IESE Business School, ha mostrado la falta de realidad de un modelo muy usado, y enseñado, de valoración de activos (Fernandez, 2015) y ha cuestionado la ética de seguir enseñándolo (Fernandez, 2019). Algo similar ocurre en otros campos, como el comportamiento humano, donde en muchas escuelas se sigue enseñando, como si fuera cierta, la pirámide de Maslow, cuando la investigación ha mostrado que es imposible diferenciar las cinco necesidades planteadas por el modelo y además sabemos que Maslow ni siquiera propuso la famosa pirámide (Bridgman, Cummings y Ballard, 2019).

\section{"Podríamos estar en camino a una visión más humana de la}

organización. Así y todo, cabe preguntarse si esto es una respuesta

\section{a la situación inmediata, y pasada la pandemia volveremos a lo habitual, o si habrá un cambio más profundo y sostenido en el tiempo en la educación en negocios"}

Estos ejemplos son muestra de un gran problema. A través de la investigación tenemos una idea mucho más completa y clara de la administración que lo que se enseña en la mayor parte de las escuelas de negocios. La investigación en administración (o management) lleva más de un siglo y nos ha permitido desarrollar una ciencia social (aún en construcción) amplia, que se nutre de otras ciencias y mantiene un vínculo permanente con la realidad.

Muchos creen que la ciencia de la administración se quedó en la "administración científica", planteada por Frederick W. Taylor, a comienzos del siglo XX (Taylor, 1911). En su modelo, Taylor planteaba un modelo mecanicista de empresa, basado en la división del trabajo y la búsqueda de eficiencia. Pero Taylor está mucho más cercano a la ingeniería que al management. Taylor es el anti-management, es decir, no necesitas hacer management porque la planificación del trabajo (realizada por ingenieros externos) y la tecnología lo harán por ti. Es importante reconocer que Taylor sí tuvo mucho éxito en el mundo real, en la práctica de empresa, ya que fue tremendamente efectivo en aumentar la eficiencia y productividad de las empresas. El problema es que lo hizo a un alto costo, la deshumanización de ellas.

Pero el estudio de la administración se hizo cargo de ello. Ya en la década de 1920 (es decir unos diez años después de la publicación de Taylor), Elton Mayo tomó la dirección de un gran proyecto de investigación que había empezado antes intentando validar las propuestas de Taylor. Sin embargo, Mayo pudo ver que no sólo no se validaban, sino que los elementos humanos aparecían como más potentes que los del trabajo en sí. Así surgía la escuela de las Relaciones Humanas, que consideraba a las personas en las empresas como parte de un sistema social.
En la siguiente década, específicamente en 1938, Chester Barnard escribió una completa teoría de las organizaciones que sentó la base para las obras de Herbert Simon (1947) y Peter Drucker (1954), entre otros. En dicha teoría, Barnard explicaba cómo las organizaciones surgen naturalmente para poder lidiar con las limitaciones propias del ser humano. EI elemento que explica entonces el surgimiento de las organizaciones es la cooperación entre personas. El considerar a la cooperación como elemento central de las organizaciones permite entender por qué la motivación, la comunicación, el trabajo conjunto y la preocupación por los demás son vitales en las organizaciones. Más aún, Barnard deja muy claro por qué las organizaciones que perduran son aquellas que buscan propósitos colectivos y potentes, mucho más allá de maximizar las utilidades de sus dueños.

Las siguientes décadas han permitido profundizar muchos aspectos de esta investigación, permitiendo conocer cómo funcionan las organizaciones y cómo poder trabajar con diferentes factores de ellas. Lamentablemente, también hemos observado una excesiva especialización de la investigación, donde cada disciplina de la administración (estrategia, comportamiento humano, finanzas, operaciones, etc.) pareciera operar desconectada de las demás. Adicionalmente, la investigación comúnmente aceptada en el circuito académico ha sido casi exclusivamente con foco en Estados Unidos, tanto en su conceptualización como en su trabajo de campo. Por ejemplo, Brewster, Gooderham y Mayrhofer (2016) muestran que 12 de los 16 artículos más citados sobre administración de recursos humanos están basados en muestras sólo de Estados Unidos. Esto ha dejado fuera por años pensamientos diferentes que pueden ampliar la mirada a las organizaciones y su administración, además de asumir que todos los fenómenos se vivirán de igual manera en distintas par- 
tes del mundo. No es raro que Ghoshal, profesor de una escuela inglesa, fuera de origen indio.

Adicionalmente, se han instalado "verdades" sin sustento, que han sido difundidas en forma acrítica por muchas escuelas de administración. Una de estas "verdades" instaladas es que el propósito de una empresa es "maximizar las utilidades de los accionistas". Esta forma de entender la empresa, coherente con los falsos supuestos denunciados por Ghoshal (2005), profundiza en la deshumanización de la empresa, sólo cambiando la técnica, promovida por Taylor (1911) por los resultados económicos. Al aceptar esto como un dogma incuestionable, se deja poco lugar para la cooperación como base de la organización, como planteaba Barnard (1938).

Pero no todo es negativo, muchas escuelas de negocio y muchos empresarios y ejecutivos han mirado la empresa en forma amplia, más allá de las utilidades que generan. Yo tuve la suerte de estudiar mi doctorado en una escuela de negocios donde leímos a Barnard, a Ghoshal, a Weick y a otros grandes autores, cuestionamos los supuestos y buscamos hacer investigación relevante que dé espacio a una mirada más comprehensiva. Tengo también la suerte de trabajar hoy en una escuela que fomenta una mirada integral y humana al mundo de las empresas. Después del 18 de octubre, realizamos un ejercicio interesante: convocamos a empresarios y ejecutivos exalumnos para discutir sobre los desafíos que planteaba para el mundo de la empresa el estallido social. A mí me correspondió codirigir una sesión sobre el propósito de la empresa. Entre los participantes se estableció un consenso acerca de la búsqueda de resultados que vayan más allá de las utilidades para los dueños o accionistas y que deben hacerse cargo de impactos en los trabajadores, en la sociedad y en el medioambiente. Además, se compartieron múltiples experiencias donde se muestra cómo estos elementos se pueden compatibilizar e incluso reforzar unos a otros. Fue muy refrescante comprobar cómo en múltiples empresas, pequeñas, medianas y grandes, empresarios y ejecutivos comprenden los problemas humanos asociados a una organización y se entregan por completo para intentar resolverlos.

Este fenómeno se está observando con creciente importancia en el mundo empresarial. Lo muestra así el movimiento mundial de empresas $B$, que es particularmente fuerte en Chile. Incluso, ya el año pasado el Business Roundtable, que agrupa a los CEOs de las principales empresas a nivel mundial, modificó sus estatutos, estableciendo que el propósito de las empresas va mucho más allá de las utilidades de sus accionistas y se relaciona con el impacto en la sociedad. Como decía Ghoshal, quizás la solución para tener una mirada más integral y humana no sea incorporar un curso más a los programas de las escuelas de negocio, sino eliminar (o modificar) parte de lo que estamos enseñando. Es fundamental que las escuelas de administración y negocios estén cerca del mundo real, observando lo bueno que se hace y aprendiendo de ello, para transmitir esos aprendizajes a los alumnos que vendrán.

Durante esta pandemia, mucho se ha hablado de una nueva perspectiva, de un cambio en la forma de entender el mundo. Las personas, viviendo una realidad muy diferente a la que acostumbraban, han podido darse cuenta de lo realmente importante en sus vidas y han descubierto la importancia de ciertas empresas y organizaciones. Las organizaciones existen para lograr satisfacer las necesidades de las personas y el management existe

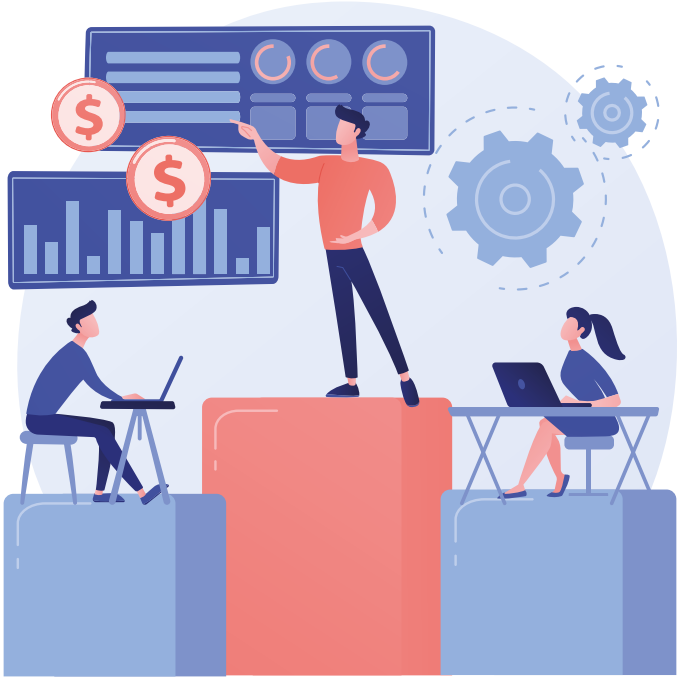

para lograr que esas organizaciones puedan cumplir con ese propósito y satisfacer las necesidades tanto de sus integrantes (para que quieran cooperar) como sus clientes o usuarios. Cuando sólo basamos la búsqueda de colaboración en lo extrínseco y sólo fijamos como objetivo lo económico quitamos el aspecto humano a la empresa, la alejamos de su esencia y, por lo tanto, quebramos su relación con la sociedad. La empresa deja de tener sentido. Sin embargo, si volvemos a la esencia de lo planteado por Barnard hace más de 80 años, entendemos que las personas, la colaboración y el propósito son los elementos centrales de la organización. Quizás no se trata de reinventar la rueda, sino de volver a lo esencial. Así, podemos encontrar respuestas a problemas nuevos. El problema no es la "administración" o el "management", sino la versión limitada que ha sido abrazada por muchas escuelas de administración en el mundo.

Referencias

Barnard, C. I. (1938). Thefunctions of the executive. Cambridge, MA: Harvard University.

Brewster, C., Gooderham, P. N. and Mayrhofer, W. (2016). Human resource management: The promise, the performance, the consequences. Journal of Organizational Effectiveness: People and Performance, 3(2), 181190.

Bridgman, T., Cummings, S., \& Ballard, J. (2019). Who built Maslow's pyramid? A history of the creation of management studies' most famous symbol and its implications for management education. Academy of Management Learning \& Education, 18(1), 81-98.

Drucker, P. F. (1954). The practice of management. New York: Harper \& Brothers.

Fernandez, P. (2015). CAPM: an absurd model. Business Valuation Review, 34(1), 4-23.

Fernandez, P. (2019). Is it ethical to teach that beta and CAPM explain something? Available at SSRN 2980847

Ghoshal, S. (2005). Bad management theories are destroying good management practices. Academy of Management Learning \& Education, 4(1), 75-91.

Kahneman, D., Knetsch, J. L., \& Thaler, R. H. (1991). Anomalies: The endowment effect, loss aversion, and status quo bias. Journal of Economic Perspectives, 5(1), 193-206.

Mayo, E. (1933). The Human Problems of An Industrial Civilization. New York: The Viking Press.

Simon, H. A. (1947). Administrative behavior. A study of decision-making processes in administrative organization. New York: Free Press.

Taylor, F. W. (1911). Scientific management. Happer \& Bros. Publishers. 